\title{
The Chromomeres of Pre-pachytene Chromosomes
}

\author{
L. F. La Cour and B. Wells \\ John Innes Institute, Colney Lane, Norwich, \\ Great Britain
}

Received August 28, 1969

\section{Introduction}

It is now well established that the synaptinemal complex (Moses 1958) is involved in pairing of homologous chromosomes at zygotene/pachytene and genetic exchange (see ref. in Moses 1968). A characteristic feature of the complex, in both plants and animals, is that most of the DNA in each of the paired homologues is confined to the chromomeres and lateral to the paired axial cores, so that recombinational DNA is segregated to the pairing space between cores. In other words, the bulk of the chromosomal DNA is not directly involved in genetic exchange. The first visible evidence of a segregation of the non-recombinational DNA is reflected in the specific chromomere pattern laid down at leptotene. It is also clear that in order to provide a pairing face, the leptotene chromomeres should be eccentric in respect of the chromosome axis, but this has yet to be shown with the light or electron microscope. The present study is concerned largely with this facet of meiotic chromosome structure.

\section{Materials and methods}

Anthers were taken from the following plants: Tulbaghia violacea, Vagaria parviflora, Fritillaria, lanceolata, the triploid lily Formobel and bulbs of the garden tulip Mad. Lefeber.

For studies with the light microscope, anthers (or strings of pollen mother cells from the lily and tulip) were fixed in acetic ethanol ( $1: 3 \mathrm{v} / \mathrm{v})$ for $4-6 \mathrm{hr}$ at $4^{\circ} \mathrm{C}$ and stored overnight or longer in $70 \%$ ethanol at $4^{\circ} \mathrm{C}$. Squash preparations were then made mostly as follows: single anthers (or strings of cells) were floated at the edge of a drop of 9 parts $2 \%$ aceto orcein : 1 part $\mathrm{N} / \mathrm{HCl}$ in a cavity slide which was heated gently by passing over a spirit flame, until the drop just steamed. The cavity slide was kept covered in a petri dish for $5 \mathrm{~min}$, in order to allow the tissues to stain. The stained tissue was then placed in a drop of $2 \%$ aceto orcein on a slide, covered with a cover slip and flattened by gentle fingertip pressure. The best results were usually obtained from preparations in which slight overstaining was corrected by rapidly running $45 \%(\mathrm{v} / \mathrm{v})$ acetic acid under the cover slip. A few conventional squash preparations were also made after pre-fixation as above, using aceto carmine or Feulgen. 


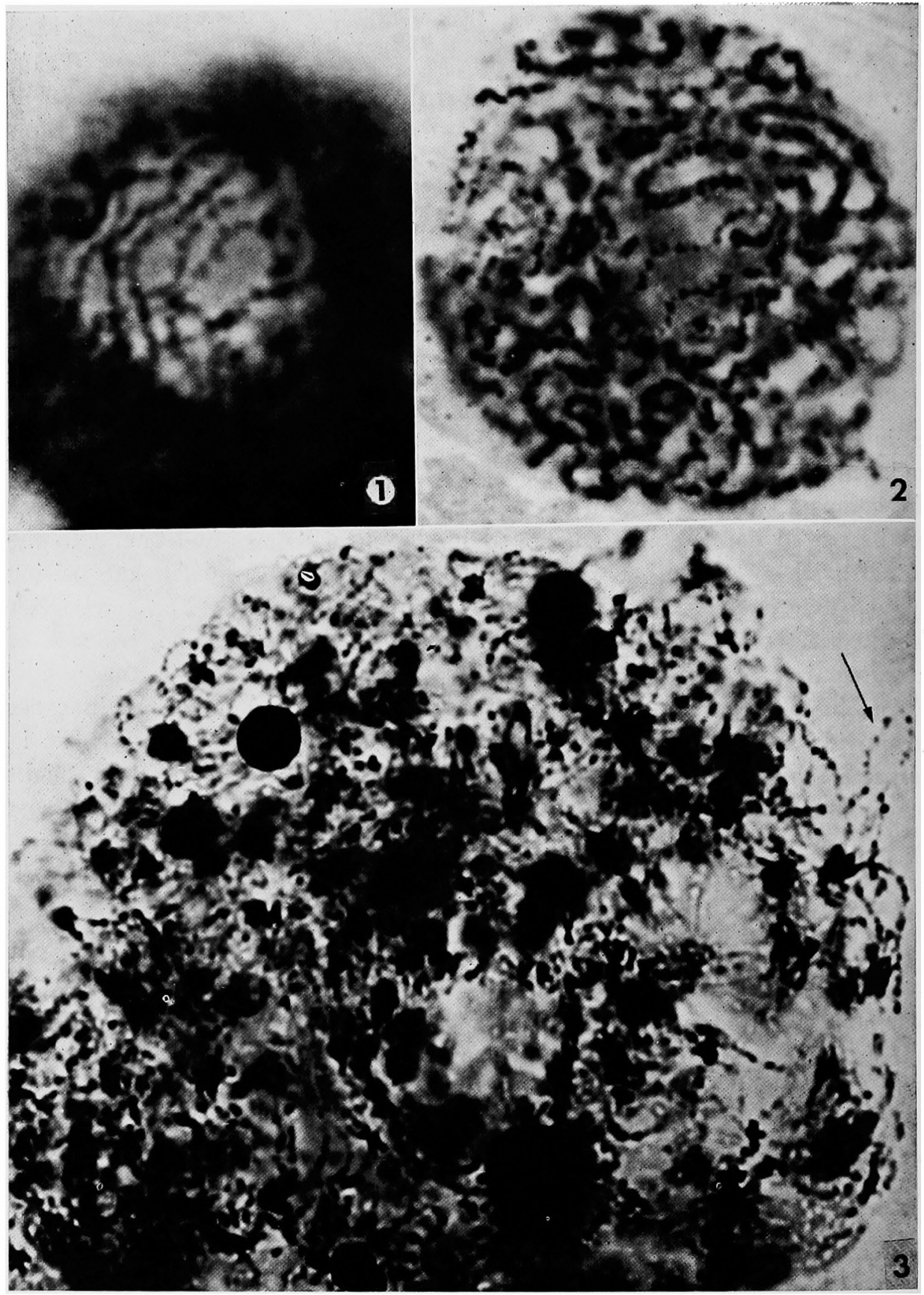

Figs. 1-3. 1, leptotene chromosomes overlying the nucleolus in a pollen mother cell from the tulip Mad. Lefeber. Note in one chromosome the eccentric arrangement of the chromomeres and their alternation around the chromosome axis. Stained in aceto orcein. $\times 2,000$. 2, zygotene nucleus from an anther of Vagaria parviflors. Note eccentric arrangement of chromomeres in the two unpaired chromosomes overlying the nucleolus in centre. Stained in aceto carmine. $\times 1,800$. 3, part of a leptotene nucleus in a pollen mother cell of Fritillaria lanceolata, showing large spherical chromocentres derived from fusion of heterochromatic segments, and eccentric arrangement of chromomeres indicated by arrow. Stained in aceto orcein. $\times 1,200$. 
For studies with the electron microscope, anthers of $T$. violacea and strings of pollen mother cells expelled from the larger anthers of the other plants were fixed in ice-cold $10 \% \mathrm{v} / \mathrm{v}$ formalin in phosphate buffer $\mathrm{pH} 7.6$ plus $0.1 \% \mathrm{w} / \mathrm{v}$ calcium chloride and $0.3 \% \mathrm{w} / \mathrm{v}$ sucrose for $18-24 \mathrm{hr}$. After fixation, the tissues were rinsed a few times in water and then transferred to Caulfield's osmium-sucrose (plus calcium chloride as above) for $2 \mathrm{hr}$, in order to enhance contrast of the image in the electron microscope. After fixation the specimens were rinsed in water and then dehydrated through a water/ethanol series. To obtain further contrast, they were stained $1 \mathrm{hr}$ in $2 \%$ uranyl acetate in absolute ethanol as the last step in dehydration. Some anthers of Tulbaghia not post-treated with osmic acid were stained for $2 \mathrm{hr}$ in $2 \% \mathrm{w} / \mathrm{v}$ phosphotungstic acid in absolute ethanol (Gordon and Bench 1968) and then rinsed briefly in absolute ethanol before pre-soaking in methacrylate. Cross-linked methacrylate (Kushida 1961) was used for embedding. Sections were cut with a Cambridge-Huxley microtome, mounted supported on "Parlodion" and then stained in lead citrate where desirable. All the electron micrographs were obtained with an Elmiskop 1a.

\section{Observations}

Light microscope: The study of leptotene and zygotene nuclei with the light microscope is impeded by the much extended condition of the chromosomes and hence their close packing in the nucleus. At best, it was possible to record photographically only parts of chromosomes at the periphery of the nucleus or stretches running over the surface of the nucleolus when this was near the surface of the nucleus.

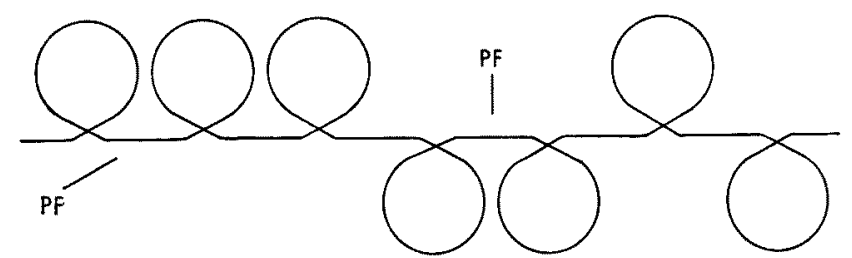

Fig. 4. Diagram illustrating the alternation of eccentric chromomeres around the axis of a leptotene chromosome, as a consequence of rotation. For simplicity the chromomeres are shown of equal size. $\mathrm{PF}=$ pairing face.

Evidence that chromomeres are indeed formed eccentrically to the axis of leptotene chromosomes is clearly demonstrated in Figs. 1-3. The eccentricity was most marked when the chromomeres are large, as in leptotene chromosomes of the tulip Mad. Lefeber (Fig. 1). A reflection of the rotation of the chromosome axis, revealed by the alternation of chromomeres to opposite sides, is apparent in the same nucleus and in stretches of two unpaired chromosomes overlying the nucleolus in the zygotene nucleus of Vagaria parviflora (Fig. 2). The effect of rotation is shown diagramatically in Fig. 4. 

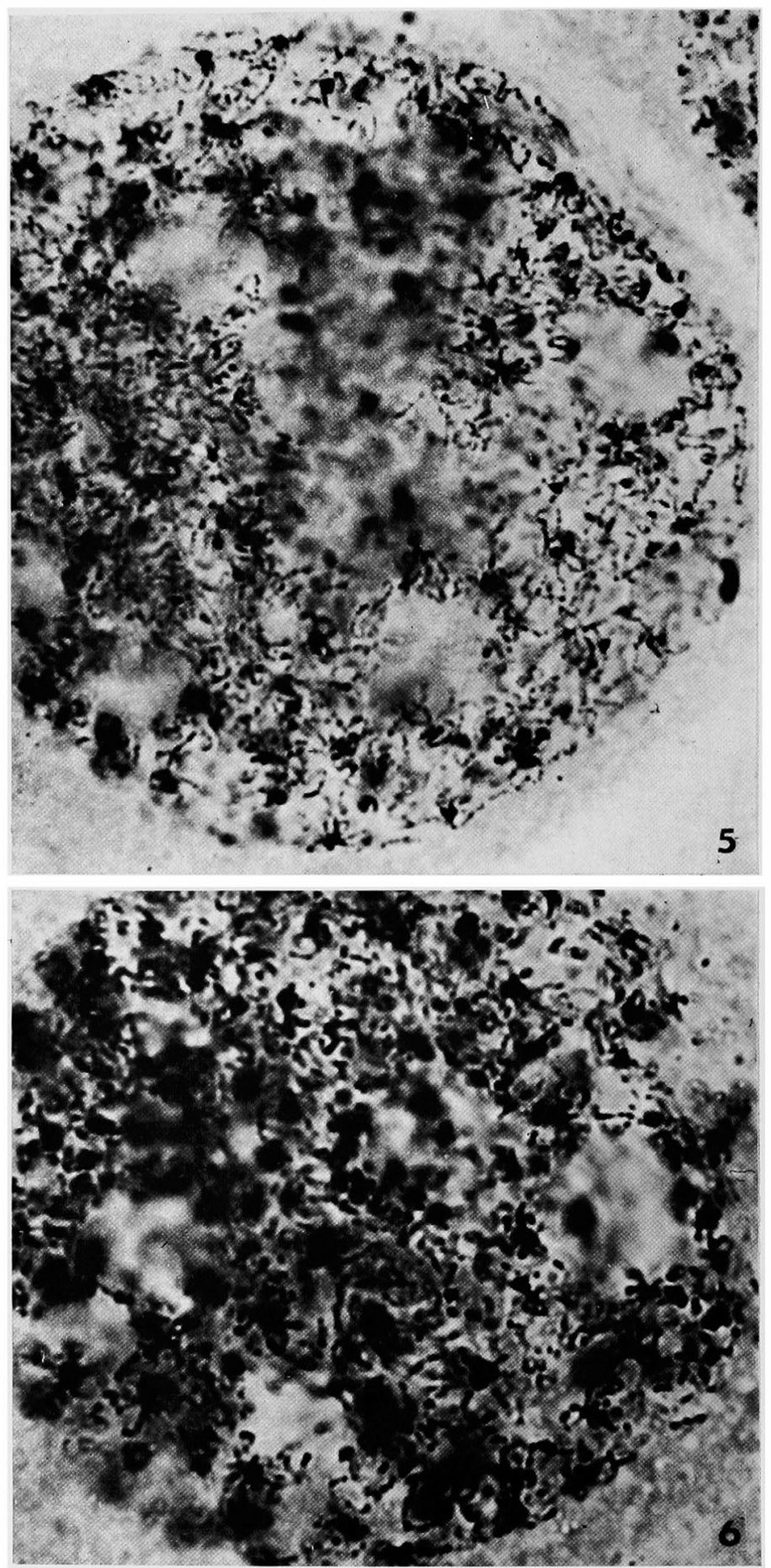

Figs. 5-6. 5, nucleus at early leptotene from an anther of the lily Formobel. The highly chromatic regions seen in Fig. 6 have mostly disappeared. Aceto orcein. $\times 1,000$. 6, premeiotic interphase nucleus from an anther of the lily Formobel. Note highly chromatic regions of chromosomes. Stained aceto orcein. $\times 1,000$. 
Fig. 5 illustrates the very beginning of leptotene in the lily Formobel, just as the chromomeres are beginning to appear. Cells at this phase of development were found together with cells at premeiotic interphase (Fig. 6) in the same loculus. It can be seen that the interphase nuclei contain a number of highly chromatic regions, identifiable as parts of chromosomes, which evidently start to disappear with the onset of leptotene. The number of such regions per interphase nucleus was difficult to estimate, but it was certainly greater than the number of chromosomes. We assume that such centres represent condensed segments of chromosomes, but regard them as distinct from other heterochromatic regions, such as those observed in $F$. lanceolata (Fig. 3) and some other plants. They were present also in nuclei in root tips and were Feulgen-positive.

It was possible to obtain better spreading of the leptotene chromosomes in Feulgen squashes of anthers from the plants studied, but with detriment to the chromomeres which were then scarcely visible (Fig. 7, see also Moens 1968). This appeared unlikely to be due to loss of material during hydrolysis in $\mathrm{N} / \mathrm{HCl}$, since a similar result was obtained after

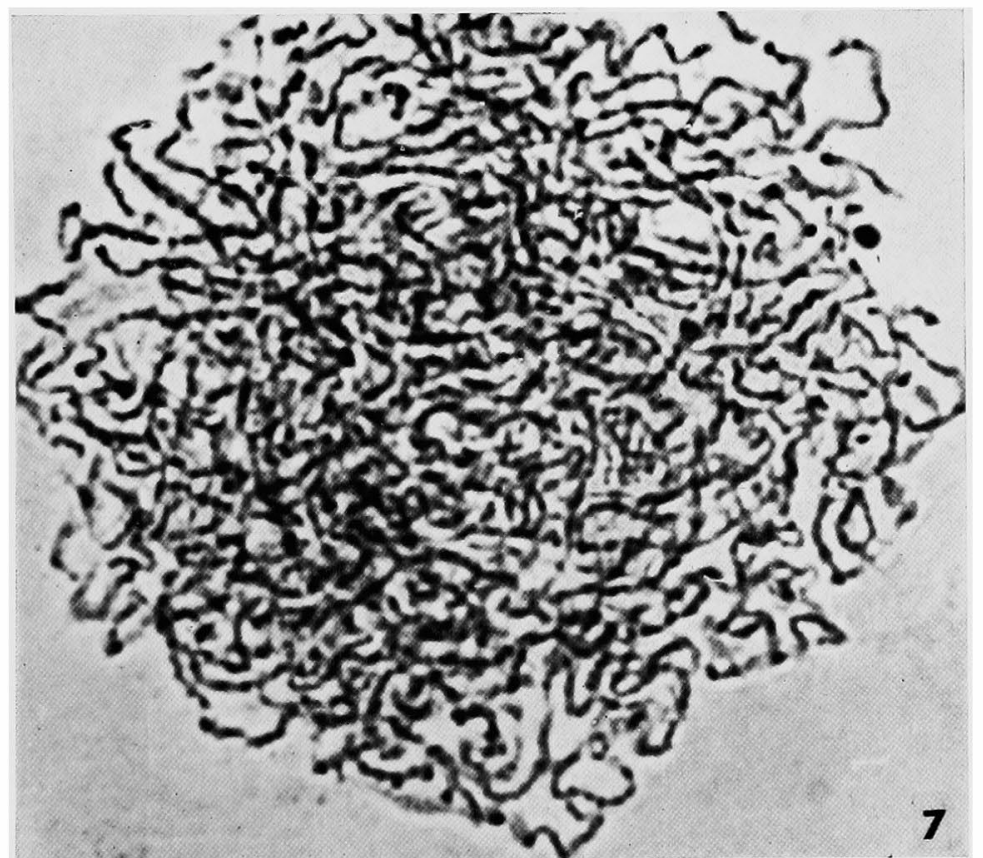

Fig. 7. Leptotene nucleus from an anther of Vagaria parviflora. The chromomeres are barely discernible in the flattened nucleus stained in Feulgen. $\times 2,000$.

treatment for a few hours in ice-cold $45 \%$ (v/v) acetic acid. The most likely explanation is that it is an effect produced by excessive flattening of cells softened by acid treatment.

Electron microscope: Previous studies with the electron microscope, in both plant and animal cells, have shown that leptotene chromosomes have dense axial cores (see ref; in Moses 1968). This was equally evident in sections of nuclei double-stained with uranyl acetate-lead citrate (Figs. 8 and 9) and in those of nuclei stained solely in ethanolic phosphotungstic acid 
(PTA), as in Fig. 10. The selectivity of PTA is attributed largely to staining of basic proteins (Bloom and Aghajanian 1966), probably by binding with lysine and arginine residues (Sheridan and Barrnett 1969).

We may note that the nucleolus, also intensly stained with PTA (Fig.

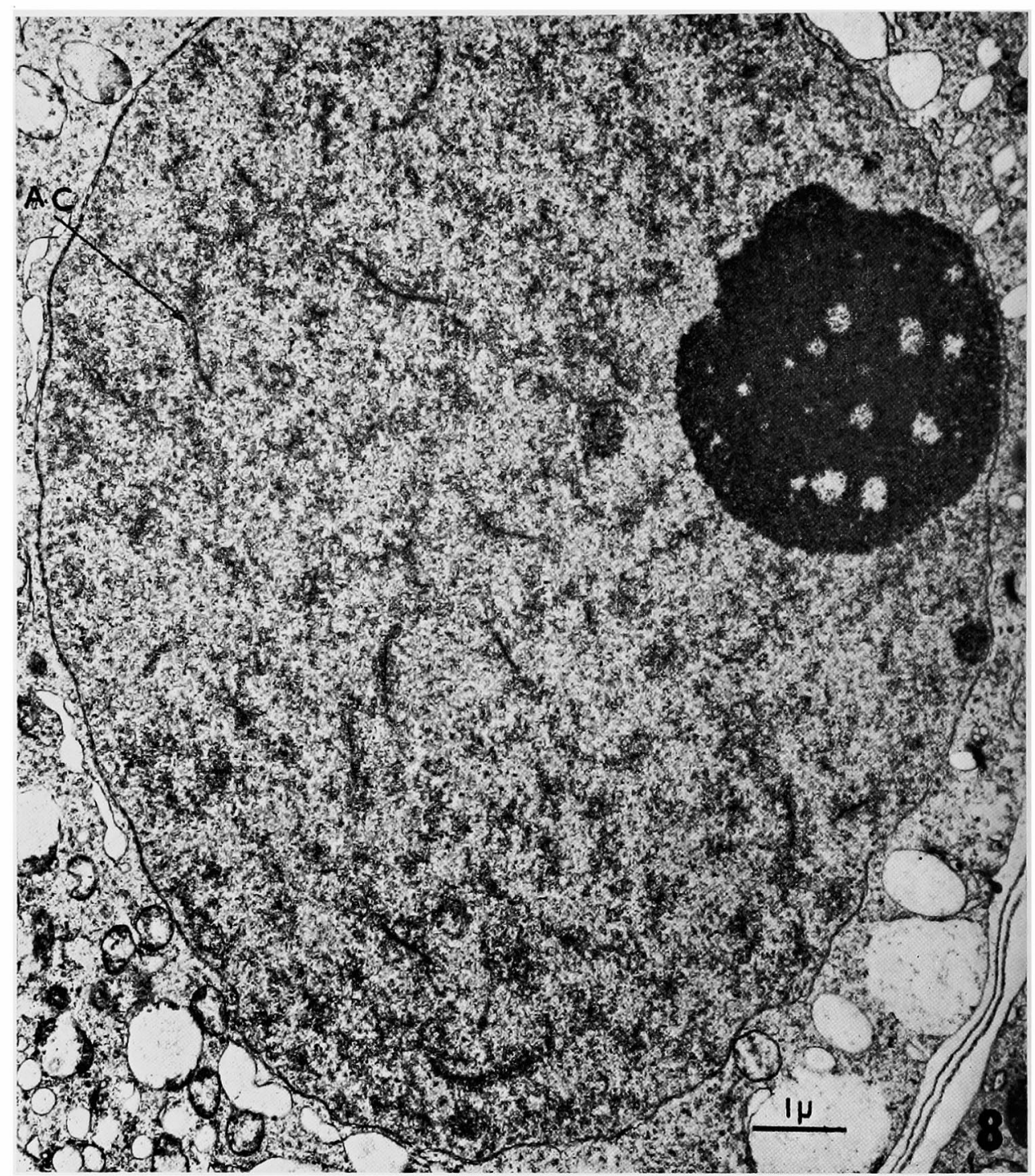

Fig. 8. Electron micrograph of a thin section of a leptotene nucleus from an anther of Tulbaghia violacea. The chromosomes have dense axial cores (AC). Stained in uranyl/lead. $\times 12,000$.

10), lies free at leptotene in Tulbaghia and not pressed against nuclear membrane to take the forms of a cap, as in lilies and some other plants (Therman 1951, cf. Moens 1968).

At leptotene the chromatin flanking the cores was more diffuse than at pachytene and the fibrils merged with less opaque ones in the nuclear sap. 
Because of this, the chromomeres were sometimes difficult to visualize at high magnification. It will be evident that in thin sections, and from con-

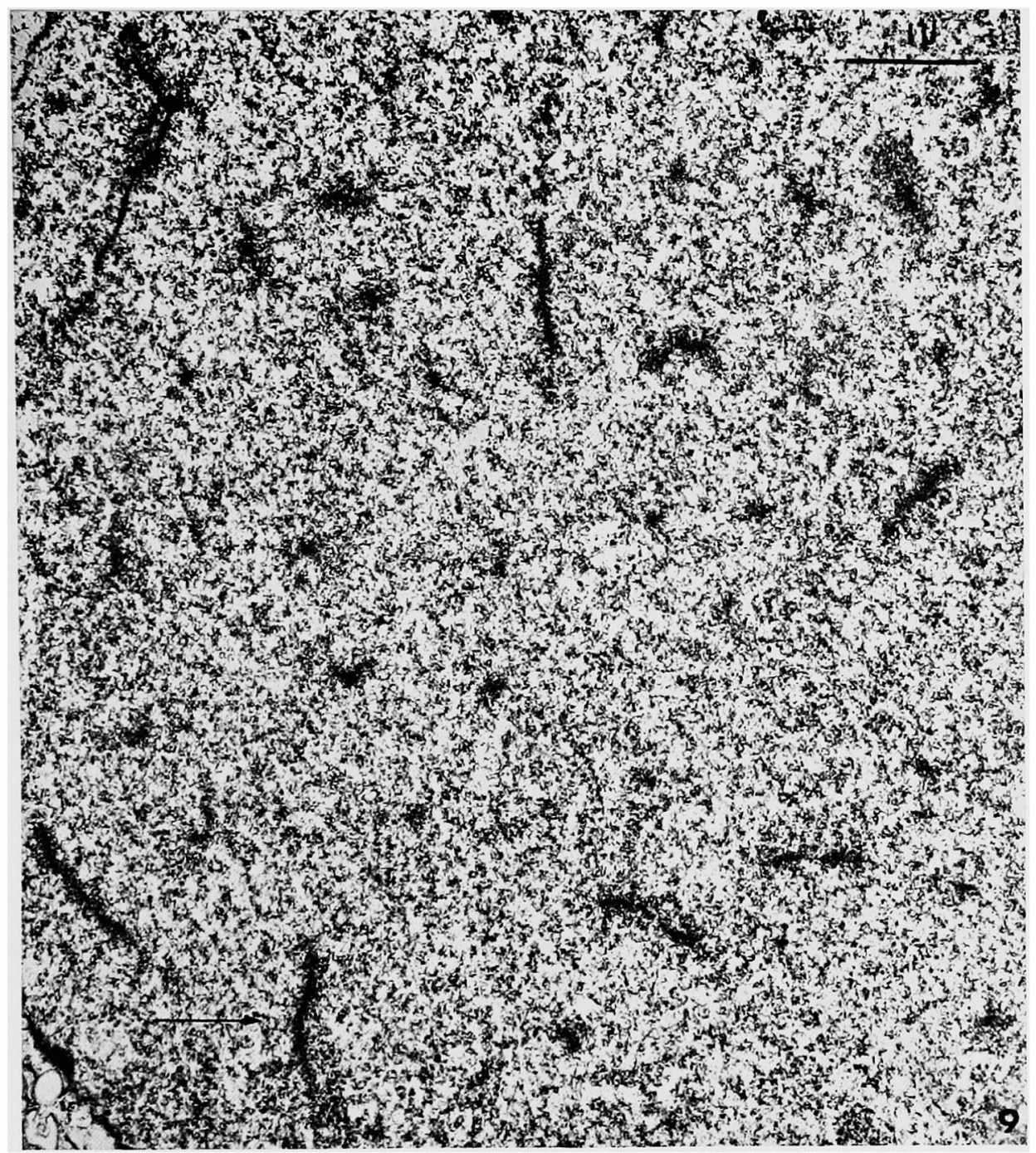

Fig. 9. Electron micrograph of a thin section of part of a leptotene nucleus from an anther of the lily Formobel. A probable example of the eccentric arrangement of chromomeres indicated by arrow. Stained uranyl/lead. $\times 16,000$.

sideration of the effect of rotation of the chromosome axis (Fig. 4), the visualization of the eccentric placement of the chromomeres was extremely difficult. Probable examples of eccentricity are shown in Figs. 9 and 11.

We examined many sections of leptotene nuclei from some of our plants without finding evidence of a close association of cores, which could be at. tributed to pre-alignment of homologous chromosomes in pairs. 


\section{Discussion}

The segregation of non-recombinational DNA from the pairing face of zygotene/pachytene chromosomes, initiated by the arrangement of the chromomeres and the specific pattern laid down at leptotene, can be interpreted as

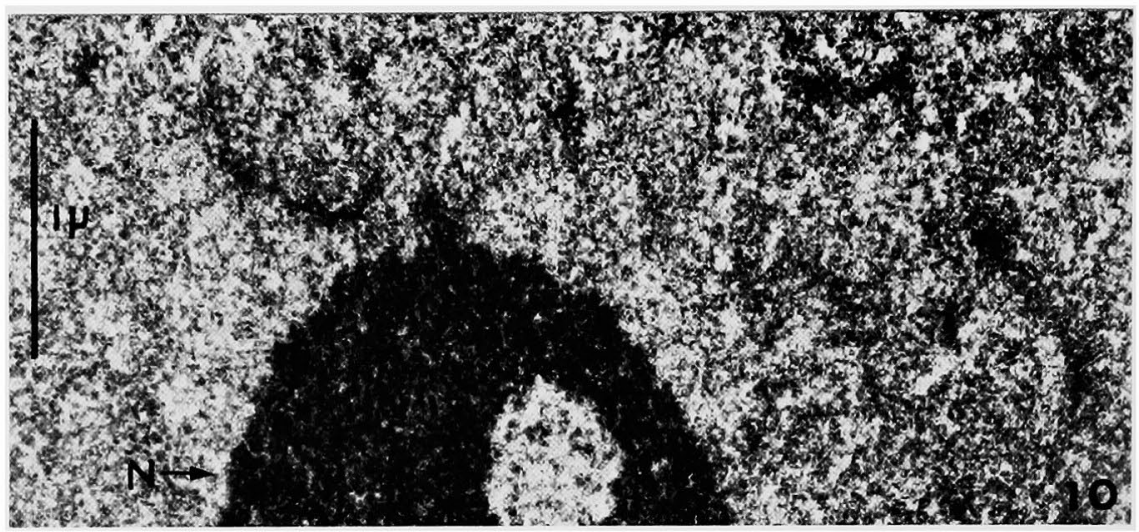

Fig. 10. Electron micrograph of a thin section of part of a leptotene nucleus from an anther of Tulbaghia violacea. Note intense staining of axial cores and nucleolus (N) after staining with ethanolic PTA. $\times 24,000$.

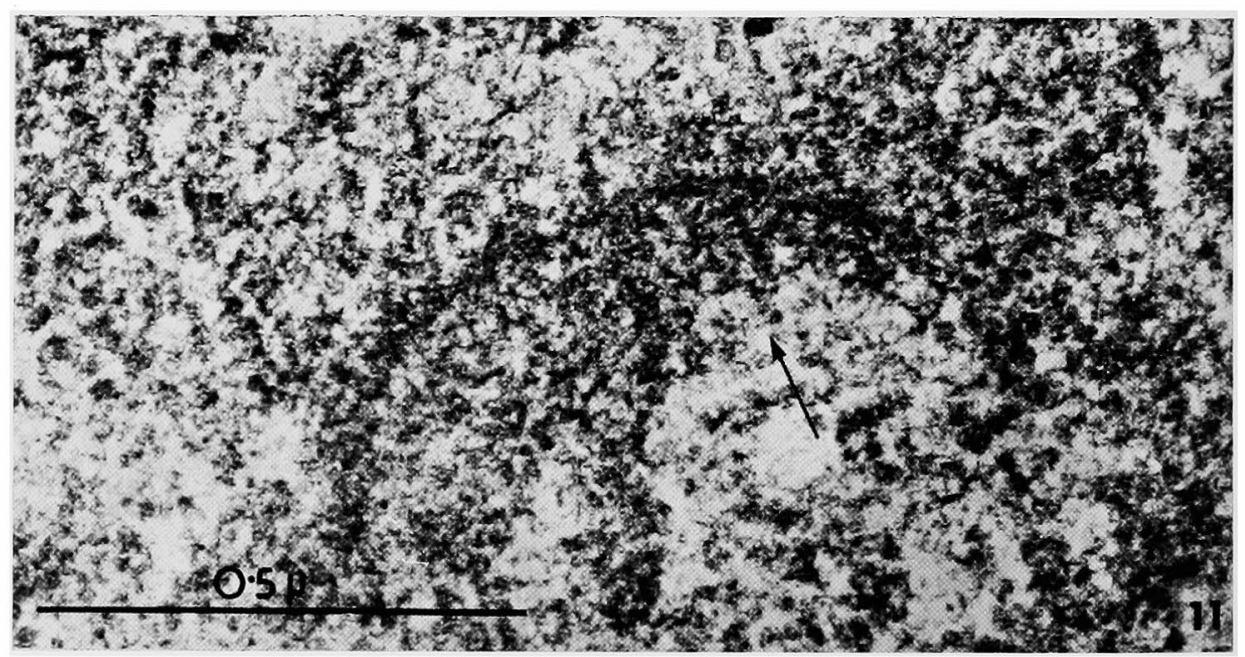

Fig. 11. Electron micrograph illustrating a probable example of the eccentric arrangement of chromomeres in leptotene chromosomes. Thin section from an anther of Vagaria parviflora stained in uranyl/lead. $\times 100,000$.

providing support for the master/slave concept proposed by Callan (1967). The eccentric character of the chromomeres is envisaged by Whitehouse (1967) in a cycloid model for the chromosome. It is logical to assume that this eccentricity derives from rotation of the chromosome axis. The super-coiled nature of the leptotene chromomeres, first seen by Ris (1957), no doubt results from this process. It probably continues until zygotene when the chromo- 
somes have already become shorter. The chromomeres are larger at pachytene.

Obviously the prominence of the cores in leptotene chromosomes cannot be due to DNA alone. The results obtained from staining leptotene chromosomes of a lily with PTA (Sheridan and Barrnett 1969) and those obtained here with similar staining of Tulbaghia chromosomes suggests that some of the opacity may be due to histone.

Although we found no evidence of a close association of leptotene cores which would support a thesis of an alignment of homologous chromosomes in pairs at the last pre-meiotic telophase, this does not mean that some degree of association does not exist. If pairing is initially confined to the centromere or chromosome ends, this would be difficult to detect in thin sections without the aid of serial sections through an entire nucleus.

Highly chromatic chromosomal regions such as were seen in pre-leptotene nuclei of a lily have been observed in similar nuclei of several plant genera (see Hiraoka 1941) and of Luzula campestris (Brown 1954). No information was given as to whether they occurred also in nuclei of root tips. Similar chromatic centres were observed by Darlington and La Cour (1946) in preleptotene nuclei of Fritillaria, where it was less certain that they were parts of chromosomes. They were stained more intensely by Feulgen than the large chromocentres derived from fusion of heterochromatic segments. No meaningful discussion can be made about these regions until more is known about their incidence in other plants and nuclei of other tissues.

\section{Summary}

The arrangement of the chromomeres in relation to the chromosome axis in pre-pachytene chromosomes was studied with the light microscope in these plants: Vagaria parviflora, Fritillaria lanceolata, the garden tulip Mad. Lefeber and the hybrid lily Formobel. The fine structure of the leptotene chromosomes of some of these was also studied with the electron microscope.

Evidence in support of the premise that chromomeres are formed eccentric to the axis of leptotene chromosomes, in order to segregate non-recombinational DNA from a pairing face, was obtained with the light microscope.

The uncoiling of condensed parts of chromosomes, seen in pre-meiotic interphases and somatic nuclei of a lily, coincided with the onset of leptotene.

Because of the alternation of chromomeres around the axis of the leptotene chromosomes, as a consequence of rotation, their eccentric alignment was difficult to confirm in thin sections with the electron microscope. The dense axial cores and less opaque chromomeral chromatin of leptotene chromosomes showed an equivalent staining behaviour with uranyl/lead ions and ethanolic phosphotungstic acid, thought to be largely specific for basic protein. No evidence was found of a close association of cores, indicative of pre-alignment 
of homologous chromosomes in pairs.

\section{References}

Callan, H. G. 1967. The organization of genetic units in chromosomes. J. Cell Sci. 2: 1-7.

Bloom, F. E. and Aghajanian, G. K. 1966. Cytochemistry of synapses: selective staining for electron microscopy. Science 154: 1575-1577.

Brown, S. W. 1954. Mitosis and meiosis in Luzula campestris D. C. Univ. Calif. Pub. Bot., 27: 231-278.

Darlington, C. D. and La Cour, L. F. 1946. Nucleic acid and the beginning of meiosis. Nature, Lond. 157: 875.

Gordon, M. and Bensch, K. G. 1968. Cytochemical differentiation of the guinea pig sperm flagellum with phosphotungstic acid. J. Ultrastruct. Res. 24: 33-50.

Hiraoka, T. 1941. Studies of mitosis and meiosis in comparison III. Behaviour of chromonemata in the pre-leptotene stage in meiosis. Cytologia 11: 473-482.

Kushida, H. J. 1961. A new embedding method for ultrathin sectioning using a methacrylate resin with three-dimensional polymer structure. J. Electronmicroscopy 10: 194.

La Cour, L. F. 1951. Heterochromatin and the organisation of nucleoli in plants. Heredity 5: $37-50$.

Moens, P. B. 1968. The structure and function of the synaptinemal complex in Lilium sporocytes. Chromosoma (Berl.) 23: 418-451.

Moses, M. J. 1958. The relation between the axial complex of meiotic prophase chromosomes and chromosome pairing in a salamander (Plethodon cinereus). J. Biophys. Biochem. Cytol. 4: 633-638.

- 1968. Synaptinemal Complex. A. Rev. Genet. 2: 363-412.

Ris, H. 1957. Chromosome structure. In The Chemical Basis of Heredity. Edit. by D. McElroy and B. Glass, Baltimore, John Hopkins Press pp. 23-69.

Sheridan, W. F. and Barrnett, R. J. 1969. Cytochemical studies on chromosome ultrastructure. J. Ultrastruct. Res. 27: 216-229.

Therman, E. 1951. Cap nucleoli in the embryo-sac mother cells of triploid Lilium tigrinum. Arch. Soc. Zool.-Bot. Fennicae 'Varama' 6: 12-17.

Whitehouse, H. L. K. 1967. A cycloid model for the chromosome. J. Cell Sci. 2: 9-22. 